\title{
Preliminary experience with pancreatic sphincterotomy as treatment for intraductal papillary mucinous neoplasm- associated recurrent pancreatitis
}

\section{다)(1) $\odot$}

\author{
Authors \\ Laura Bernardoni", Stefano Francesco Crinò , Giorgia De Conti, Maria Cristina Conti Bellocchi, Nicolò De Pretis, \\ Antonio Amodio, Luca Frulloni, Armando Gabbrielli
}

Institution

Gastroenterology and Digestive Endoscopy Unit, The Pancreas Institute, University of Verona, G.B. Rossi

University Hospital, Verona, Italy

submitted 23.3.2017

accepted after revision 31.7.2017

\author{
Bibliography \\ DOI https://doi.org/10.1055/s-0043-119753 | \\ Endoscopy International Open 2017; 05: E1144-E1150 \\ (c) Georg Thieme Verlag KG Stuttgart · New York \\ ISSN 2364-3722
}

\section{Corresponding author}

Stefano Francesco Crinò, MD, Gastroenterology and

Digestive Endoscopic Unit, G.B. Rossi University Hospital,

P. le L.A. Scuro 10, Verona - 37134, Italy

Fax: +39-45-8124898

stefanofrancesco.crino@aovr.veneto.it

\section{ABSTRACT}

Background and study aims Pancreatic intraductal papillary mucinous neoplasms (IPMN) are cystic tumors of the pancreas characterized by a malignant potential. IPMN have been associated with recurrent pancreatitis (RP). Obstruction of the main pancreatic duct by thick mucus has been postulated to be the cause of pancreatitis. In a few isolated reports, pancreatic sphincterotomy (PS) has been reported to reduce the frequency of pancreatitis. The aim of this study was to assess the efficacy of PS in patients with IPMN-associated RP.

Patients and methods We retrospectively identified patients with RP and IPMN who underwent PS from June 2010 to December 2015. Patients were included in two different groups: (a) main duct/mixed type IPMN (MD-IPMN) and (b) branch duct IPMN (BD-IPMN) with or without worrisome features/high risk stigmata. Other causes of RP were excluded. The number of pancreatitis episodes occurring during a comparable time period before and after PS was evaluated.

Results In total, 16 patients were analyzed (seven included in the MD-IPMN group and nine in the BD-IPMN group). The numbers of pancreatitis episodes occurring before and after PS were $3.5 \pm 2.32$ and $0.56 \pm 1.03$, respectively $(P<$ 0.0001). Mean follow-up was 27.4 months (range 6-63 months). Complete, partial (reduction of pancreatitis episodes $>50 \%$ ), and no response were obtained in 11 (68.7\%), 3 (18.7\%), and 2 patients (12.5\%), respectively. One $(6.25 \%)$ case of mild post-endoscopic retrograde cholangiopancreatography (ERCP) pancreatitis was observed. No cancer was detected in resected patients. None of the BD-IPMN group patients had or developed worrisome features/high risk stigmata during follow-up.

Conclusions PS seems to be effective in reducing the number of episodes of IPMN-associated pancreatitis and should be considered as a treatment option in selected clinical settings. However, active surveillance should be continued considering the malignant potential of IPMN. Further prospective controlled studies are needed to confirm our results.

\section{Introduction}

Intraductal papillary mucinous neoplasms (IPMN) are common pancreatic cystic tumors characterized by a pathological proliferation of mucin-producing epithelial cells with an increased risk of pancreatic cancer. Overproduction of mucus results in

\footnotetext{
* Drs Bernardoni and Crinò contributed equally to this article.
}

cystic or saccular dilations of the pancreatic ductal system. IPMN may involve the branch ducts (BD-IPMN), the main duct (MD-IPMN), or both (mixed-IPMN) [1]. IPMN is suspected to cause acute or recurrent pancreatitis (RP) in $7-67 \%$ of cases [2-4]. Obstruction of the pancreatic duct (PD) by highly viscous mucus is postulated to determine the development of pancreatitis. 
IPMN-associated RP may worsen a patient's quality of life and progresses to chronic pancreatitis. Moreover, some authors have suggested a higher risk of malignancy in IPMN causing acute pancreatitis. Therefore, pancreatitis may indicate the need for surgical resection even when there are no signs of malignancy by imaging or cytology as reported in the Fukuoka consensus [5]. However, pancreatic surgery has a high incidence of complications and mortality even when performed at high volume centers. Moreover, surgical risks are increased in elderly patients with comorbidities.

Based on the postulated IPMN-associated pancreatitis pathophysiology, we hypothesized that pancreatic sphincterotomy (PS) facilitates mucus outflow into the duodenum, reduces the intraductal pressure, and reduces pancreatitis episodes. However, to date, only a few isolated cases examining this hypothesis have been reported [6-8]. We conducted a retrospective study to assess the safety and efficacy of PS in reducing the frequency of IPMN-associated pancreatitis.

\section{Patients and methods}

\section{Study approval and patient population}

This study was approved by the Verona University Hospital Ethics Committee (Prot.n.5006).

We retrospectively selected patients with IPMN diagnosis and RP who underwent PS from June 2010 to December 2015 using our prospectively collected endoscopic retrograde cholangiopancreatography (ERCP) database.

Diagnosis of IPMN was achieved by:

1. Cytology after endoscopic ultrasound-guided fine-needle aspiration, when performed.

2. MRI with magnetic resonance cholangiopancreatography (MRCP) with or without secretin injection and/or endoscopic ultrasound (EUS). Imaging criteria used to diagnose IPMN were: (a) presence of one or more dilated branch ducts $(\geq 10 \mathrm{~mm})$ communicating with a non-dilated $(<5 \mathrm{~mm}) \mathrm{PD}$ (BD-IPMN); (b) presence of a segmental or diffusely dilated $\mathrm{PD}$ ( $\geq 5 \mathrm{~mm}$ ) with no dilation of secondary ducts (MD-IPMN); (c) presence of a dilated PD ( $\geq 5 \mathrm{~mm}$ ) communicating with one or more dilated branch ducts (mixed-IPMN).

3. ERCP findings: (a) spontaneous extruding mucus from the papilla ("fish-eye"); (b) mucus extrusion from the papillary orifice after sphincterotomy or intraductal injection of contrast medium; (c) mucinous filling defects in the main pancreatic duct (linear filling defect during low pressure pancreatography); (d) cystic pancreatic branch duct opacification.

Definitive differential diagnosis between MD-IPMN and chronic pancreatitis (CP) was achieved by previously described ERCP findings (points [a] and/or [b] and/or [c]).

Acute pancreatitis was defined as abdominal pain associated with three times more than the upper normal limit for serum pancreatic enzymes (i.e. amylases or lipases) and/or pancreatitis at imaging (CT or MRI). RP was defined as having at least two episodes of pancreatitis that resulted in hospital admission during the last 2 years.

\section{Exclusion criteria}

Patients with other causes of recurrent pancreatitis were excluded: (a) suspected biliary etiology (episodic jaundice and/or transient elevation of liver function tests and/or evidence of gallbladder/choledochal stones or sludge at MRI or EUS [9]); (b) alcohol use (>40 g/day [10]); (c) smoking (>12 pack-years [11]); (d) pancreas divisum with santorinicele at MRI [12]; (e) autoimmune pancreatitis according to the International Consensus Diagnostic Criteria [13]; (f) hypertriglyceridemia (>1000 mg/dL) [14]; (g) associated gene mutations (CFTR, SPINK1, PRSS1, CTRC) and/or with a family history of pancreatitis [15]; (h) consumption of medication associated with acute pancreatitis [16]; (i) presence of CP changes at MRI [17] and/ or at endoscopic pancreatography ( $\geq$ grade 2 according to the Cambridge classification [18]).

Furthermore, patients with less than 6 months follow-up after PS were excluded.

\section{Outcome measures}

Epidemiological data, IPMN features (type, location, and size), ERCP findings and related adverse events, and the number of pancreatitis episodes that occurred before and after endoscopic treatment were recorded for each patient.

We assessed the number of pancreatitis episodes that occurred before PS using historical medical documentation provided by patients at the time of hospitalization.

Pancreatitis episodes occurring after PS were assessed both by reviewing the medical records of the scheduled follow-up visits and by phone contact in all patients. At our institution, patients treated with PS for IPMN-associated pancreatitis are actively followed by medical visits or phone contact (based on patients preference) 6 months after the procedure and then yearly. In both cases, we investigated abdominal pain recurrence, any emergency room access, or hospitalization or laboratory/imaging examination performed after PS. The medical documentation provided by the patients was analyzed and acute pancreatitis episodes fulfilling the previously reported criteria were recorded.

We defined "complete response" as the absence of pancreatitis during follow-up. A "partial response" was defined as a reduction $>50 \%$ of pancreatitis episodes after PS.

Patients were classified into two groups: MD-IPMN (including mixed type IPMN) and BD-IPMN (with or without worrisome features).

\section{Endoscopic procedures}

All endoscopic procedures were performed under general anesthesia with a lateral-viewing duodenoscope (TJF-160 and TJF180; Olympus, Tokyo, Japan). Hyoscine-butylbromide (40 mg) was injected intravenously to inhibit duodenal peristalsis. Deep cannulation of the PD was achieved by a standard sphincterotome both over the wire or by injection of contrast medium; after pancreatography, a longest possible (based on the anatomical landmark) guidewired selective pancreatic sphincterotomy was performed. An ERBE EndoCut electroincision was used in all patients. No plastic stent was inserted because of the high risk of occlusion. 
- Table 1 Demographic and clinical features of patients analyzed.

\begin{tabular}{|c|c|}
\hline No. of patients & 16 \\
\hline Age, mean (range), years & $61(41-84)$ \\
\hline Sex, male/female & $13 / 3$ \\
\hline \multicolumn{2}{|l|}{ Indication for PS, n (\%) } \\
\hline - MD-IPMN unfit for/refusing surgery & $7(44 \%)$ \\
\hline - BD-IPMN without worrisome feature & $9(56 \%)$ \\
\hline \multicolumn{2}{|l|}{ Type of IPMN, n (\%) } \\
\hline - MD-IPMN & $2(13 \%)$ \\
\hline - BD-IPMN & $9(56 \%)$ \\
\hline - Mixed-IPMN & $5(31 \%)$ \\
\hline \multicolumn{2}{|c|}{ Location (and MD mean size) of MD/mixed-IPMN, n (\%) } \\
\hline - Head $(6.5 \mathrm{~mm})$ & $6(86 \%)$ \\
\hline - Body & $0(0 \%)$ \\
\hline - Tail & $0(0 \%)$ \\
\hline - Diffuse (7 mm) & $1(14 \%)$ \\
\hline \multicolumn{2}{|l|}{ Location (and mean size) of BD-IPMN, $n$ (\%) } \\
\hline - Head-Uncinate (13 mm) & $8(89 \%)$ \\
\hline - Body (10 mm) & $1(11 \%)$ \\
\hline - Tail & $0(0 \%)$ \\
\hline \multicolumn{2}{|c|}{$\begin{array}{l}\text { PS, pancreatic sphincterotomy; MD-IPMN, main duct/mixed type intraductal } \\
\text { papillary mucinous neoplasm; BD-IPMN, branched duct intraductal papillary } \\
\text { mucinous neoplasm. }\end{array}$} \\
\hline
\end{tabular}

\section{Statistical analysis}

Continuous variables were assessed for normality and expressed as means and standard deviation (SD). Paired Student's $t$ test was used to compare the mean values of variables before and after treatment. Categorical variables were expressed as frequencies with percentages. The chi-squared test with Yates' correction was used for categorical data. All tests were twotailed. Fisher's exact test was used in a $2 \times 2$ table where applicable. Statistical significance was determined by a $P$ value $<0.05$. Data were analyzed using SPSS 22 software (SPSS, IBM, Chicago, United States).

\section{Results}

In total, 26 IPMN patients suffering from RP who underwent PS were identified. Of these, 10 patients were excluded: 1 was lost at follow-up, 2 were affected by pancreas divisum with santorinicele, 3 had signs of chronic pancreatitis, and 4 did not reach 6 months of follow-up after PS because they underwent pancreatic resection. Therefore, 16 patients (13 men and 3 women with a mean age of 60.7 years [range $41-84$ years]) were included in our study and analyzed ( $>$ Table 1 and $\triangleright$ Fig. 1).

Seven patients were included in the MD-IPMN group and nine patients were included in the BD-IPMN group. All MD-IPMN pa-

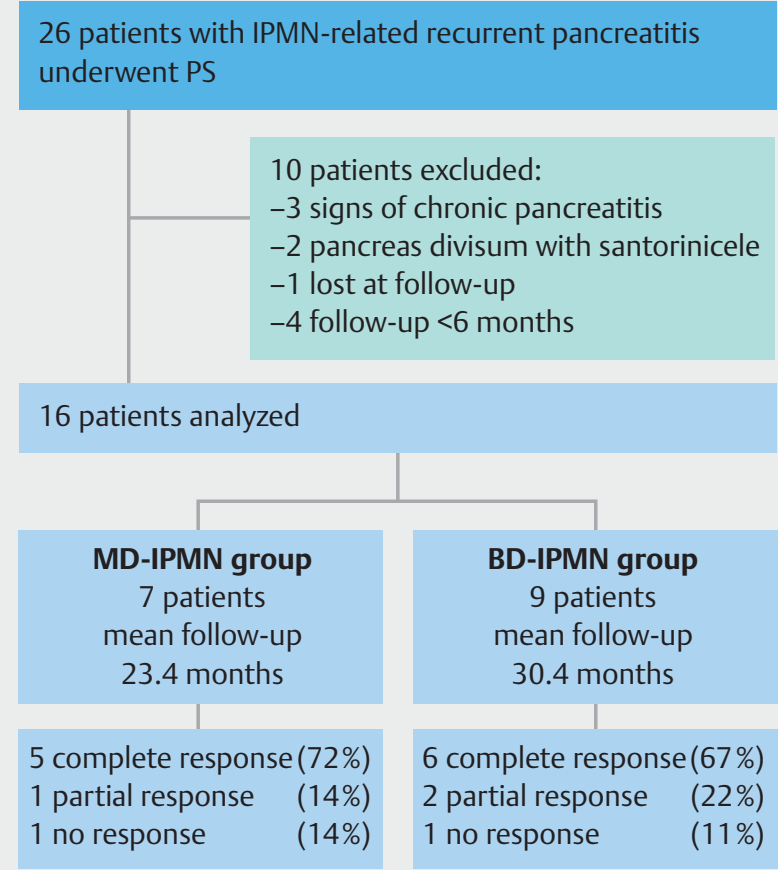

- Fig. 1 Study flow chart of the patients analyzed. PS, pancreatic sphincterotomy. MD-IPMN, main duct/mixed type intraductal papillary mucinous neoplasm; BD-IPMN, branched duct intraductal papillary mucinous neoplasm.

tients underwent PS because severe comorbidities ( $\geq$ ASA IV according to the American Society of Anesthesiology's Classification) precluded surgery or patients refused resection. No high risk stigmata/worrisome features were observed by imaging according to the Fukuoka consensus [5] in patients with BD-IPMN.

ERCP findings were: spontaneous extruding mucus from the papilla sign in 2 patients (the papilla orifice was not widely dilated and PS was normally performed); mucus extrusion from the papilla after PS in 4; PD dilation without strictures and cystic branch duct opacification in 4; normal PD and cystic branch duct opacification in 4; and normal pancreatography in 3 ( $\triangleright$ Table 2). PS was performed at major papilla in 15 patients and at minor papilla in 1 patient with a concomitant pancreas divisum. No procedure-related mortality was observed. One patient $(6.25 \%)$ experienced a mild post-ERCP pancreatitis that was managed within 3 days with medical therapy.

The mean follow-up for the study population was 27.4 months (range 6-66 months). The average number of pancreatitis episodes that occurred during the same interval of time before and after PS were $3.5 \pm 2.32$ and $0.56 \pm 1.03$, respectively $(P<0.0001)$.

The mean follow-up times for the MD-IPMN and BD-IPMN groups were 23.4 months and 30.4 months, respectively. The average number of pancreatitis episodes that occurred during a period of time equal to the follow-up before and after endoscopic treatment in the MD-IPMN and BD-IPMN groups were $3 \pm$ 1.41 and $0.57 \pm 1.13(P=0.015)$, and $3.89 \pm 2.85$ and $0.56 \pm 1.01$ $(P=0.014)$, respectively ( $\triangleright$ Table 3 and $\triangleright$ Fig. 2$)$. 
Table 2 Main ERCP findings of the 16 patients analyzed stratified by IPMN type.

\begin{tabular}{|c|c|c|c|}
\hline & MD-IPMN $(n=2)$ & Mixed-IPMN $(n=5)$ & BD-IPMN $(n=9)$ \\
\hline Spontaneous extruding mucus from the papilla & & 2 & \\
\hline Mucus extrusion after PS & 1 & 1 & 2 \\
\hline PD mucinous filling defects ${ }^{1}$ & 1 & & \\
\hline PD dilation without strictures + cystic branch duct opacification & & 3 & \\
\hline Cystic branch duct opacification & & & 3 \\
\hline Normal & & & 3 \\
\hline
\end{tabular}

- Table 3 Primary outcome and follow-up of whole population and in the three groups. Time before pancreatic sphincterotomy (PS) was calculated in each single patient based on the length of the maximum follow-up available. Time before and after PS, were therefore identical in every single patient.

\begin{tabular}{|c|c|c|c|c|}
\hline & \multicolumn{2}{|c|}{ Number of pancreatitis episodes (mean \pm SD) } & \multirow[t]{2}{*}{$P$ value ${ }^{1}$} & \multirow[t]{2}{*}{ Follow-up mean (range), months } \\
\hline & Before PS & After PS & & \\
\hline All patients analyzed & $3.50 \pm 2.32$ & $0.56 \pm 1.03$ & $<0.0001$ & $27.4(6-63)$ \\
\hline MD-IPMN & $3 \pm 1.41$ & $0.57 \pm 1.13$ & 0.0149 & $23.4(6-48)$ \\
\hline BD-IPMN & $3.89 \pm 2.85$ & $0.56 \pm 1.01$ & 0.0142 & $30.4(7-63)$ \\
\hline
\end{tabular}

PS, pancreatic sphincterotomy; MD-IPMN, main duct/mixed type intraductal papillary mucinous neoplasm; BD-IPMN, branched duct intraductal papillary mucinous neoplasm.

1 Paired $t$ test.
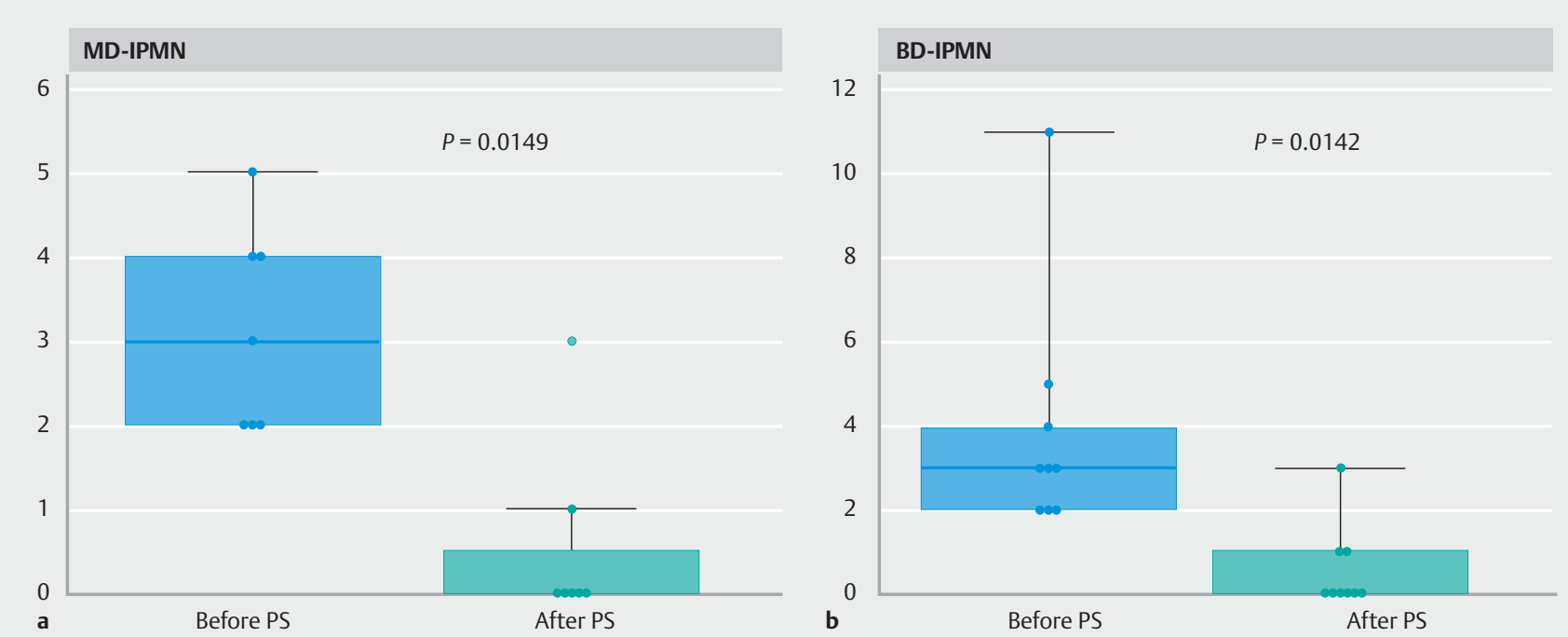

Fig. 2 Number of pancreatitis episodes (ordinate) calculated in a comparable time before and after pancreatic sphincterotomy (PS). a MD-IPMN group $(n=7)$; b BD-IPMN group $(n=9)$. $P=$ paired $t$ test. MD-IPMN, main duct/mixed type intraductal papillary mucinous neoplasm; BD-IPMN, branched duct intraductal papillary mucinous neoplasm.

A complete response was observed in 11 patients $(68.7 \%$, mean follow-up 24.9 months, range 6 - 66 months). A partial response was found in three patients (18.7\%, mean follow-up 40.6 months, range 11-63 months). These three patients experi- enced only one episode of pancreatitis during the follow-up. For comparison, these patients had five, four, or three pancreatitis episodes that occurred during an equivalent period of time before PS. No response was observed in two patients $(12.5 \%$, 
- Table 4 Correlation between clinical outcomes, type of IPMN and dilation of the main duct. Mean follow-up of responders and not responders after PS.

\begin{tabular}{|c|c|c|c|}
\hline & Responders $^{1}$ & Not responders & $P$ value $^{2}$ \\
\hline $\mathrm{n}(\%)$ & $14(88 \%)$ & $2(12 \%)$ & \\
\hline \multicolumn{4}{|l|}{ Type of IPMN } \\
\hline - BD-IPMN & $8(57 \%)$ & $1(50 \%)$ & 1 \\
\hline - MD-IPMN & $6(42 \%)$ & $1(50 \%)$ & \\
\hline \multicolumn{4}{|l|}{ Main duct dilation } \\
\hline . 5-9mm & $6(78 \%)$ & $1(100 \%)$ & \\
\hline - $\geq 10 \mathrm{~mm}$ & $1(22 \%)$ & 0 & 1 \\
\hline Follow-up, mean (range) & $28.2(6-66)$ & $21(16-26)$ & - \\
\hline
\end{tabular}

mean follow-up 21 months, range $16-26$ months). These two patients experienced three pancreatitis episodes each during the follow-up compared with three and two episodes occurring during an equivalent period of time before PS.

Two patients underwent surgical resection during follow-up:

- One patient with no response after PS in the MD-IPMN group underwent total pancreatectomy 16 months after sphincterotomy. This patient became fit for surgery because of a significant clinical improvement after a cerebral vascular accident. Histology showed high grade dysplasia of the main duct epithelium.

- One patient from the BD-IPMN group underwent pyloruspreserving pancreatoduodenectomy after 26 months because of a suspicion of main duct involvement and no response to PS. Histology showed low grade dysplasia of the ductal epithelium.

IPMN diagnosis was confirmed in both resected patients and no invasive carcinoma was observed histologically.

No statistically significant difference was found between response and type of neoplasm (BD-IPMN vs. MD-IPMN) $(P=1)$. Furthermore, no relationship was found between response and dilation of the PD $(5-9 \mathrm{~mm}$ vs $\geq 10 \mathrm{~mm})(P=1)$ ( $\triangleright$ Table 4$)$.

Interestingly, none of the BD-IPMN group patients had or developed worrisome features/high risk stigmata during follow-up. On the other hand, follow-up data were not available for the MD-IPMN group patients because surveillance was stopped due to their unfit-for-surgery condition.

\section{Discussion}

The results of our preliminary study suggest that PS is effective in preventing pancreatitis relapses in patients with IPMN and RP. In the literature, only six cases of IPMN-associated pancreatitis successfully treated with PS are described [6-8]. In 1998, Elton et al. [7] reported three cases where PS resulted in the resolution of symptoms in "mucinous ductal ectasia" patients.
More recently, Oh and Dua [6] described two cases of IPMNrelated RP, both of which improved after PS.In the present study, complete or partial responses were observed in $85 \%$ of patients during a mean follow-up of 22 months.

PS has been proposed for patients with MD-IPMD or BDIPMN. For MD-IPMN, obstruction of the PD by mucus is largely accepted $[19,20]$. Obstruction of the PD by thick mucus leads to ductal hypertension, which is responsible for premature release and activation of pancreatic enzymes. This same mechanism has also been postulated for BD-IPMN; in these cases, mucin is suspected to migrate into the main duct [21]. The efficacy of PS in BD-IPMN seems to confirm this hypothesis. However, our study's patient population was limited in size and type. Therefore, we cannot draw any definitive conclusions because this sample population was not representative of all IPMN patients. PS clearly does not change the IPMN-associated risk of cancer and survival. Therefore, the aim of performing PS in patients unfit for surgery and in patients with BD-IPMN without worrisome features/high risk stigmata is to improve patient's quality of life and to avoid pancreatic surgery in patients without malignant features at imaging, respectively. Interestingly, four of the excluded patients were treated with "bridge to surgery" aim. While these patients wait for treatment by tertiary care centers with long waiting lists, a high frequency of pancreatitis may increase the risk of delaying surgery even further. PS may prevent or reduce the recurrence of pancreatitis which would allow the centers to perform surgery at the scheduled time.

However, the most important result from our study was observed in patients with BD-IPMN who had no evidence of PD involvement/high risk stigmata/worrisome features at imaging. Indeed, these patients should undergo surgical resection even in the absence of any signs of malignancy, according to international guidelines [5]. This is particularly relevant in cases involving older patients or where lesions are located in the pancreatic head, requiring duodenopancreatectomy. PS seems to be an effective treatment for this group of patients because we ob- 
served complete or partial responses in $89.9 \%$ of patients. In other words, PS should reduce the need for resective pancreatic surgery in patients with BD-IPMN; however, pancreatitis may be a sign of malignancy, and surgery may be required regardless of the presence of high risk stigmata/worrisome features. However, the association between pancreatitis and malignant IPMN is still debated. Pancreatitis seems to increase the risk for malignancy in retrospective surgical series, even for BD-IPMN $[22,23]$; however, the findings from preoperative imaging are not reported in these studies and the presence of other potential causes of pancreatitis (e.g. alcohol, chronic pancreatitis, etc.) was not excluded. Indeed, the risk for malignancy does not seem to increase in one medical series as well as in two other surgical studies after exclusion of other causes of pancreatitis $[2,24,25]$. In our series, the nine patients in the BDIPMN group did not have any evidence of high risk stigmata/ worrisome features. Only one of these patients underwent surgery for involvement of PD and this patient's surgical specimen showed histological evidence of low grade dysplasia. The remaining eight patients did not show any morphological changes during a mean follow-up of 31 months and they are still under active radiologic surveillance. Further studies are needed to confirm that pancreatitis does not increase the risk of malignancy in BD-IPMN patients in the absence of high risk stigmata/worrisome features.

In the present study, all BD-IPMN were located in the head of the pancreas. Therefore, we may assume that the mucus produced by the neoplasm may easily migrate from the involved branch duct to the pre-papillary PD leading to obstruction. However, no definitive data are available and this hypothesis should be considered to be purely speculative until the publication of a larger study. Indeed, IPMN of the tail seems to manifest less frequently with pancreatitis $[2,24]$ and patients with this form of IPMN undergo surgery that utilizes a laparoscopic approach that is rarely contraindicated. Moreover, we believe that PS would be less effective in patients with IPMN of the tail because it is likely that the obstruction could occur along the PD and distant from the papilla.

The major limitation of our study is the absence of a control group. However, in Jang et al. [2], recurrence of pancreatitis was observed in $50 \%$ of 10 patients with IPMN-associated pancreatitis not resected because of comorbidities. Our encouraging results must be confirmed in larger, randomized controlled trials. In conclusion, PS is safe and may be helpful in the management of select patients with IPMN-associated pancreatitis.

\section{Competing interests}

None
References

[1] Farrell JJ. Prevalence, diagnosis and management of pancreatic cystic neoplasms: current status and future directions. Gut Liver 2015; 23 : $571-589$

[2] Jang JW, Kim MH, Jeong SU et al. Clinical characteristics of intraductal papillary mucinous neoplasm manifesting as acute pancreatitis or acute recurrent pancreatitis. J Gastroenterol Hepatol 2013; 28: 731 738

[3] Venkatesh PG, Navaneethan U, Vege SS. Intraductal papillary mucinous neoplasm and acute pancreatitis. J Clin Gastroenterol 2011; 45: $755-758$

[4] Pelletier AL, Hammel P, Rebours V et al. Acute pancreatitis in patients operated on for intraductal papillary mucinous neoplasms of the pancreas: frequency, severity, and clinicopathologic correlations. Pancreas 2010; 39: 658-661

[5] Tanaka M, Fernández-del Castillo C, Adsay V et al. International consensus guidelines 2012 for the management of IPMN and MCN of the pancreas. Pancreatology 2012; 12: 183-197

[6] Oh YS, Dua K. Pancreatic sphincterotomy for pancreatitis associated with main duct intraductal papillary mucinous neoplasm. Endoscopy UCTN: 2011; 43: (Suppl. 02): E75

[7] Elton E, Howell DA, Parson WG et al. Endoscopic pancreatic sphincterotomy: indications, outcome, and a safe stentless technique. Gastrointest Endosc 1998; 47: 240-249

[8] Nocente R, Gentiloni N, Gasbarrini A et al. An apparent idiopathic case of relapsing acute pancreatitis. Hepatogastroenterology 2001; 48: $572-573$

[9] Lee SP, Nicholls JF, Park HZ. Biliary sludge as a cause of acute pancreatitis. NEJM 1992; 326: 589-593

[10] Yadav D, Whitcomb DC. The role of alcohol and smoking in pancreatitis. Nat Rev Gastroenterol Hepatol 2010; 7: 131-145

[11] Yadav D, Hawes RH, Brand RE et al. Alcohol consumption, cigarette smoking, and the risk of recurrent acute and chronic pancreatitis. Arch Intern Med 2009; 169: 1035-1045

[12] Crinò SF, Bernardoni L, Conti Bellocchi MC et al. Efficacy of endoscopic minor papilla sphincterotomy for symptomatic santorinicele. Clin Gastroenterol Hepatol 2017; 15: 303-306

[13] Shimosegawa T, Chari ST, Frulloni L et al. International consensus diagnostic criteria for autoimmune pancreatitis: guidelines of the International Association of Pancreatology. Pancreas 2011; 40: 352 358

[14] Toskes PP. Hyperlipidemic pancreatitis. Gastroenterol Clin North Am 1990; 19: $783-791$

[15] Raphael KL, Willingham FF. Hereditary pancreatitis: current perspectives. Clin Exp Gastroenterol 2016; 26: 197-207

[16] Nitsche C], Jamieson N, Lerch MM et al. Drug induced pancreatitis. Best Pract Res Clin Gastroenterol 2010; 24: 143 - 155

[17] Hansen TM, Nilsson M, Gram M et al. Morphological and functional evaluation of chronic pancreatitis with magnetic resonance imaging. World J Gastroenterol 2013; 19: 7241 - 7246

[18] Sarner M, Cotton PB. Classification of pancreatitis. Gut 1984; 25 : $756-759$

[19] Fernandez-del C, Targarona J, Thayer SP et al. Incidental pancreatic cysts: clinicopathologic characteristics and comparison with symptomatic patients. Arch Surg 2003; 138: 427 - 433 ; discussion 433-434

[20] Tanaka M, Kobayashi K, Mizumoto K et al. Clinical aspects of intraductal papillary mucinous neoplasm of the pancreas. J Gastroenterol 2005; 40: 669-675

[21] Ringold DA, Shroff P, Sikka SK et al. Pancreatitis is frequent among patients with side-branch intraductal papillary mucinous neoplasia diagnosed by EUS. Gastrointest Endosc 2009; 70: 488 - 494 
[22] Morales-Oyarvide V, Mino-Kenudson M, Ferrone CR et al. Acute pancreatitis in intraductal papillary mucinous neoplasms: A common predictor of malignant intestinal subtype. Surgery 2015; 158: 12191225

[23] Tsutsumi K, Ohtsuka T, Oda Y et al. A history of acute pancreatitis in intraductal papillary mucinous neoplasms of the pancreas is a potential predictive factor for malignant papillary subtype. Pancreatology 2010; 10: 707-712
[24] Roch AM, Parikh JA, Al-Haddad MA et al. Abnormal serum pancreatic enzymes, but not pancreatitis, are associated with an increased risk of malignancy in patients with intraductal papillary mucinous neoplasms. Surgery 2014; 156: $923-930$

[25] Pelletier AL, Hammel P, Rebours V et al. Acute pancreatitis in patients operated on for intraductal papillary mucinous neoplasms of the pancreas: frequency, severity, and clinicopathologic correlations. Pancreas 2010; 39: 658-661 